\title{
Diabetes care in pediatric refugees from Africa or Middle East: experiences from Germany and Austria based on real-world data from the DPV registry
}

\author{
Nicole Prinz ${ }^{1,2, *}$, Katja Konrad ${ }^{3,4, *}$, Christoph Brack ${ }^{5}$, Eva Hahn ${ }^{6}$, Antje Herbst ${ }^{7}$, Andrea Icks ${ }^{2,8,9}$, \\ Jürgen Grulich-Henn ${ }^{10}$, Norbert Jorch ${ }^{11}$, Christian Kastendieck ${ }^{12}$, Kirsten Mönkemöller ${ }^{13}$, Oliver Razum ${ }^{14}$, \\ Claudia Steigleder-Schweiger ${ }^{15}$, Michael Witsch ${ }^{16}$ and Reinhard W Holl, ${ }^{1,2}$ on behalf of the DPV Initiative
}

${ }^{1}$ Institute of Epidemiology and Medical Biometry (ZIBMT), University of UIm, Ulm, Germany, ${ }^{2}$ German Center for Diabetes Research (DZD), Munich Neuherberg, Germany, ${ }^{3}$ Department of Pediatric and Adolescent Medicine, University of Cologne, Cologne, Germany, ${ }^{4}$ Department of Pediatric and Adolescent Medicine, Elisabeth-Hospital Essen, Essen, Germany, ${ }^{5}$ Pediatric Practice, Celle, Germany, ${ }^{6}$ Department of Pediatrics and Adolescent Medicine, Protestant Hospital Oberhausen, Oberhausen, Germany, ${ }^{7}$ Department of Pediatric and Adolescent Medicine, Hospital Leverkusen gGmbH, Leverkusen, Germany, ${ }^{8}$ Institute for Health Services Research and Health Economics, German Diabetes Center (DDZ), Leibniz Institute for Diabetes Research at Heinrich Heine University Düsseldorf, Düsseldorf, Germany, ${ }^{9}$ Institute for Health Services Research and Health Economics, Faculty of Medicine, Heinrich Heine University Düsseldorf, Düsseldorf, Germany, ${ }^{10}$ Department of Pediatrics, University of Heidelberg, Heidelberg, Germany, ${ }^{11}$ Department of Pediatrics, Protestant Hospital Bielefeld gGmbH, Bielefeld, Germany, ${ }^{12}$ Department of Pediatrics, Clinic Bremen-Mitte, Bremen, Germany, ${ }^{13}$ Department of Pediatrics, Pediatric Hospital Amsterdamer Straße, Cologne, Germany, ${ }^{14}$ School of Public Health, Bielefeld University, Bielefeld, Germany, ${ }^{15}$ Department of Pediatrics, University Hospital of Pediatrics, Paracelsus Medical University Salzburg, Salzburg, Austria, and ${ }^{16}$ Department of Pediatric and Adolescent Medicine, Centre Hospitalier de Luxembourg, Luxembourg, Luxembourg

*(N Prinz and K Konrad contributed equally to this work)

Correspondence should be addressed to N Prinz Email nicole.prinz@uni-ulm.de

\section{Abstract}

Objective: With increasing migration to Europe, diabetes diagnosis and treatment of refugees became challenging. To describe the current experience with pediatric refugees in Germany and Austria.

Design and Methods: 43,137 patients (<21 years) with type 1 diabetes from the diabetes patient follow-up registry (DPV) were studied and divided by refugee status into patients born in Middle East $(n=365)$ or Africa $(n=175)$ and native patients (child and parents born in Germany/Austria; G/A: $n=42,597)$. Groups were compared using multivariable regression adjusted for age, sex and diabetes duration (SAS 9.4). In refugees the first year after arrival was studied, and for native children the most recent year of care.

Results: After adjustment, HbA1c was highest in refugees (1. ME and 2. AFR vs 3. G/A: $72.3 \pm 1.0$ and $75.0 \pm 1.4$ vs $66.0 \pm 0.1 \mathrm{mmol} / \mathrm{mol}, 1$ vs $3: P<0.001$ and 2 vs $3: P<0.001$ ) and microalbuminuria ( 9.9 and 13.6 vs $6.5 \%, 1$ vs 3 : $P=0.039$ and 2 vs $3: P=0.002)$ was more prevalent. African children experienced severe hypoglycemia $(17.8 \pm 4.3$ and $25.4 \pm 8.7$ vs $11.5 \pm 0.3$ per 100 patient years, 1 vs 3: $P>0.05$ and 2 vs $3: P=0.045)$ significantly more often, whereas hypoglycemia with coma $(5.1 \pm 1.1$ and $4.1 \pm 1.6$ vs $2.6 \pm 0.1$ per 100 patient years, 1 vs $3: P=0.006$ and 2 vs $3: P>0.05)$ and retinopathy ( 2.1 and n/a vs $0.2 \%, 1$ vs $3: P<0.001$ ) were significantly more common in children from Middle East compared to natives. Insulin pumps were used in a markedly larger proportion of native patients (7.4 and 13.2 vs $43.0 \%, 1$ vs $3: P<0.001$ and 2 vs 3: $P<0.001$ ).

Conclusions: A relevant number of pediatric refugees with type 1 diabetes are treated in German/Austrian diabetes clinics. Refugee children, parents and caregivers are faced with several problems in diabetes therapy and outcome that should be addressed more intensively by pediatric diabetes teams. 


\section{Introduction}

Due to the increasing number of refugees in Western and Central Europe, countries such as Germany and Austria are faced with a challenge politically, socially, and also medically. With increased migration, the number of refugees from Africa (AFR) or the Middle East (ME) with diabetes is on the rise. The majority are still coming from Syria, Iraq and Afghanistan (1), although another flow of refugees exist from North Africa, mainly the Maghreb states. Diabetes health care teams and the national health care systems are faced with the changing population structure and the increasing cultural diversity that leads for example to language problems and challenges diabetes care.

The right to health care for refugee children is codified in the UN Convention on the Rights of the Child (UNCRC) (2). It states that child refugees should have access to health services equivalent to those of the local population in the host country (2). A recent publication however found that most European countries $(80 \%)$ do not entitle migrant children to the care codified in the UNCRC (3). In our previous DPV analyses, rate of insulin pump therapy was reported to be roughly half among pediatric type 1 diabetes patients with Turkish background compared to those without migration background (4). In addition, depending on the region of origin, additional differences in diabetes therapy and outcome were present (5).

Refugee children with non-infectious chronic diseases such as type 1 diabetes and their families are in an exceptional situation after arrival in the host country. Despite laws and agreements regulating the access to health care, prejudice and uncertainty toward the social interaction with refugees exist. Real-world registry data provide a unique source to study current diabetes practice and experience among pediatric refugees. The aim of this study was to investigate diabetes care in the first year of stay in Germany/Austria among pediatric refugees from the ME and Africa and to compare the results to native patients in order to describe current diabetes treatment and outcome.

\section{Methods}

\section{Data collection}

Data were collected during routine care and retrieved from the standardized diabetes patient follow-up (DPV; 'Diabetes-Patienten-Verlaufsdokumentation', www.d-p-v.eu), a German/Austrian/Luxembourgian and
Swiss database for people with diabetes. Anonymized data are transmitted twice a year from participating health care facilities, using the DPV software, to Ulm, Germany, for central analysis $(5,6)$. Implausible and inconsistent data are reported back to the centers for correction. 182 German diabetes centers and 11 centers from Austria taking care of pediatric refugees from Africa and/or the ME contributed data to this analysis. The Ethical Committee of Ulm University has approved the DPV initiative and the institutional review boards of each participating clinic approved the anonymized data collection.

\section{Patients}

We initially included 45,400 patients ( $<21$ years) with type 1 diabetes $(n=43,137 ; 95.0 \%)$, type 2 diabetes $(n=774$; $1.7 \%)$ or other specific types of diabetes $(n=1489 ; 3.3 \%)$ in our analysis. Patients' medical data were documented between January 1995 and March 2018. Both patients and parents are asked for their country of birth. To group patients by refugee status, country of birth was used. Children born in ME (Syria, Afghanistan, Iran and Iraq) or Africa were classified as refugees. In Africa, all countries were considered; Morocco (25.4\%), Egypt (12.7\%), Eritrea (9.0\%), Libya (7.9\%), Somalia (7.9\%), Ethiopia (5.8\%) and Tunisia (5.3\%) contributed more than 5\% of African refugees, respectively. Native patients were defined as child and both parents born in Germany or Austria. In refugees, the first year of care after arrival in the host country was studied, for native patients the most recent year of care. In case of multiple visits per patient per year, data were aggregated.

\section{Diabetes care processes and outcomes}

\section{Anthropometry}

To adjust for age and sex, body mass index standard deviation scores (BMI-SDS) were calculated using national reference data from the German Health Interview and Examination Survey for Children and Adolescents (KiGGS) (7). As there are no reliable reference data for children from Africa or the ME, the KiGGS data were also applied for refugee children.

\section{Metabolic control}

Glycemic control was evaluated by HbA1c. In order to adjust for differences among laboratories, HbA1c data were mathematically standardized to the DCCT reference 
range of $20.77-42.62 \mathrm{mmol} / \mathrm{mol}(4.05-6.05 \%)$ using the multiple of the mean (MOM) method (6).

\section{Treatment modalities}

Insulin treatment regimen was classified as conventional insulin therapy (CT; 1-3 daily injection time points), intensive insulin treatment (ICT; 4-8 daily injection time points) or insulin pump therapy (CSII). Daily insulin dose per kilogram bodyweight and daily number of SMBG were studied.

\section{Acute diabetes complications}

Diabetic ketoacidosis was defined as blood $\mathrm{pH}$ value $<7.3$ or a clinical diagnosis associated with inpatient care. Severe hypoglycemic episodes were defined as self-reported cognitive impairment, being unable to take glucose, glucagon or other corrective actions without help from others. Hypoglycemia with coma was defined as a sub-group of severe hypoglycemia associated with loss of consciousness or seizure.

\section{Long-term diabetes complications}

Microalbuminuria was defined as at least two positive urine tests (spot urine, overnight collection or 24-h urine samples) within 1 year. Diabetic retinopathy (non-proliferative or proliferative) was diagnosed if patientshadatleastoneabnormal eye examination within the respective year of study. Arterial hypertension was defined as taking antihypertensive medication and/or elevated blood pressure above the 95th percentile of the KiGGS reference population (8). Dyslipidemia was diagnosed if patients were taking lipid-lowering drugs or had permanently (defined by at least two consecutive measurements) increased total cholesterol, low-density cholesterol, triglycerides (TGs) or permanently decreased high-density cholesterol. Cut-off values were as follows: total cholesterol $>5.2 \mathrm{mmol} / \mathrm{L}, \quad \mathrm{LDL}>3.4 \mathrm{mmol} / \mathrm{L}, \quad \mathrm{HDL}<0.9 \mathrm{mmol} / \mathrm{L}$ and TG $>1.5 \mathrm{mmol} / \mathrm{L}$. Moreover, number of inpatient admissions per 100 patient years was studied.

\section{Statistical analysis}

Statistical analysis was performed using the SAS statistical software package (version 9.4; SAS Institute Inc.).
All measures are presented as median with interquartile range (IQR) or as percentage. For unadjusted group comparison of continuous variables, Kruskal-Wallis test was used. Binary variables were compared by $\chi^{2}$ test. To adjust for confounding effects of sex, age and diabetes duration, multivariable regression analyses (linear regression for continuous variables, logistic regression for binary outcomes and negative binomial regression for number of events) were applied. The method of TukeyKramer was used to adjust for multiple comparisons. To investigate the impact of the region of diabetes manifestation on treatment regimen in type 1 diabetes patients, a sub-analysis on 437 refugees with additional information on the place of diabetes manifestation, i.e. abroad prior to migration vs after migration to Germany/ Austria, was performed. A two-sided $P$ value $<0.05$ was considered statistically significant.

\section{Results}

Among the pediatric patients studied, we identified 584 children (1.3\%) with African or Middle Eastern migration background, here defined as refugees. The majority of refugee children from the $\mathrm{ME}(n=272,67.3 \%)$ immigrated between 2014 and 2018, whereas most refugees from Africa $(n=137,72.5 \%)$ immigrated prior to 2014. More than half of refugee children from ME (73.4\% out of $n=338$ ) or Africa (56.7\% out of $n=120$ ) with available information on the place of diabetes manifestation were diagnosed with diabetes in their home country prior to the migration to Germany or Austria.

\section{Data on type 2 diabetes or other specific types of diabetes}

Although a relevant number of pediatric patients with type 2 diabetes $(n=774)$ or other specific types of diabetes ( $n=1489$ ) has been documented in the database, the number of refugee children born in Africa or the Middle East with type 2 diabetes (AFR: 0.9\%, $n=7$; ME: $1.7 \%$, $n=13$ ) or other specific types of diabetes (AFR: $0.3 \%$, $n=5$; ME: $1.3 \%, n=19$ ) was low. Hence, no statistical evaluation of diabetes care process and outcome was performed. Table 1 summarizes the various causes of other specific types of diabetes documented for the refugees studied. Six out of 24 patients had MODY diabetes (Table 1). 
Table 1 Different causes of other specific types of diabetes in refugee children from Africa or Middle East.

\begin{tabular}{|c|c|c|}
\hline & $\begin{array}{l}\text { Africa } \\
(n=5)\end{array}$ & $\begin{array}{c}\text { Middle } \\
\text { East }(n=19)\end{array}$ \\
\hline MODY diabetes & 1 & 5 \\
\hline Diabetes in thalassemia & - & 4 \\
\hline Diabetes in down syndrome & 1 & 2 \\
\hline Diabetes due to other genetic diseases & 2 & 2 \\
\hline Mitochondrial diabetes & - & 3 \\
\hline $\begin{array}{l}\text { Diabetes due to diseases of the } \\
\text { exocrine pancreas }\end{array}$ & - & 2 \\
\hline Drug-induced diabetes & 1 & - \\
\hline Not otherwise specified & - & 1 \\
\hline
\end{tabular}

\section{Data on type 1 diabetes}

Among the refugee children studied, 539 patients had type 1 diabetes (AFR: $n=175$, ME: $n=365$ ). Demographics and the average number of visits per year are summarized in Table 2, stratified by refugee status. The majority of patients both refugees and natives have been seen by the diabetes care team at least four times a year (ME vs AFR vs G/A: 71.0 vs 70.3 vs $73.6 \%$ ). Compared to native children, refugees from the ME or Africa were younger and had shorter diabetes duration, with lowest values in children with African background. After adjustment for age, sex and diabetes duration, BMI-SDS was similar between native children and refugees.

\section{Metabolic control and diabetes outcome}

Table 3 summarizes results of multivariable regression analyses with adjustments for age, sex and diabetes duration. Glycemic control differed significantly among groups, with highest HbA1c levels in refugees from Africa and lowest values in native children.
Compared to native patients, acute diabetes complications such as severe hypoglycemia were more common in refugees with African background, whereas refugees from the ME experienced hypoglycemia with coma significantly more often. DKA did not differ among groups.

With regard to long-term diabetes complications, microalbuminuria was present in refugees significantly more often compared to natives, whereas retinopathy was more common in children with Middle Eastern refugee status. Frequencies of dyslipidemia and hypertension did not differ among groups. Overall, the number of hospital admissions was higher in refugees (Table 3).

\section{Treatment regimen}

Treatment modalities differed between groups, with markedly higher use of insulin pump therapy in native children compared to refugees from either Africa or the ME. Intensive insulin therapy was the most common treatment modality in refugees (Fig. 1). Refugee children from the ME required more insulin per kilogram body weight compared to refugees from Africa or native patients, although the difference between refugees from the ME and Africa was of minor clinical relevance (Table 3).

Interestingly, the region of diabetes manifestation seemed to have an impact on treatment modality. Although not significant after adjustment for age, sex and diabetes duration, children with Middle Eastern refugee status used insulin pump therapy more often if diabetes was diagnosed in Germany or Austria compared to children who were diagnosed with diabetes in their home country prior to migration ( 12.0 vs $5.4 \%, P=0.09$ ). This is in contrast to refugees from Africa, where insulin pump therapy was documented more frequently in children

Table 2 Demographics of pediatric type 1 diabetes patients, stratified by refugee status. Data are expressed as non-adjusted median with interquartile range or as percentage, except for BMI-SDS. Due to multiple comparisons, $P$ values were adjusted using false discovery rate algorithm.

\begin{tabular}{|c|c|c|c|c|c|c|}
\hline & \multicolumn{3}{|c|}{ Refugee status } & \multicolumn{3}{|c|}{$P$ value } \\
\hline & 1. $\mathrm{ME}(n=365)$ & 2. $\operatorname{AFR}(n=175)$ & 3. Native $(n=42,597)$ & 1 vs 2 & 1 vs 3 & 2 vs 3 \\
\hline Girls, \% & 41.9 & 41.7 & 46.6 & ns & ns & ns \\
\hline Age, years & $11.8(8.2-15.3)$ & $12.4(8.5-15.8)$ & $15.6(12.0-17.5)$ & ns & $<0.001$ & $<0.001$ \\
\hline Diabetes duration, years & $1.8(0.4-6.0)$ & $0.6(0.3-3.6)$ & $5.2(2.3-8.9)$ & 0.006 & $<0.001$ & $<0.001$ \\
\hline BMI-SDS $^{*}$ & $0.21 \pm 0.05(n=359)$ & $0.27 \pm 0.07(n=171)$ & $0.27 \pm 0.01(n=41,960)$ & ns & ns & ns \\
\hline 1 visit per year, \% & 14.5 & 10.8 & 7.3 & ns & $<0.001$ & ns \\
\hline 2 or 3 visits per year, $\%$ & 14.5 & 18.9 & 19.1 & ns & ns & ns \\
\hline$\geq 4$ visits per year, $\%$ & 71.0 & 70.3 & 73.6 & ns & ns & ns \\
\hline
\end{tabular}

*Adjusted mean \pm S.E.M., based on multivariable linear regression model with adjustment for age, sex and diabetes duration; $P$ value adjusted for multiple comparisons using the method of Tukey-Kramer.

AFR, Africa; BMI-SDS, body mass index standard deviation score; ME, Middle East. 
Table 3 Metabolic control and diabetes complications in pediatric type 1 diabetes patients, stratified by refugee status.

\begin{tabular}{l}
\hline \\
\hline HbA1c, mmol/mol \\
HbA1c, \% \\
Insulin dose, IU/kg*day \\
SMBG, per day \\
Severe hypoglycemia, \\
per 100 pat. yrs. \\
Hypoglycemia with coma, \\
per 100 pat. yrs. \\
DKA, per 100 pat. yrs. \\
Hypertension, $\%$ \\
Dyslipidemia, $\%$ \\
Microalbuminuria, $\%$ \\
Retinopathy, $\%$ \\
Inpatient admissions, \\
per 100 pat. yrs.
\end{tabular}

\begin{tabular}{c}
\hline \\
\hline $1 . \operatorname{ME}(n=365)$ \\
\hline $72.3 \pm 1.0(n=361)$ \\
$8.77 \pm 0.09$ \\
$0.992 \pm 0.016(n=358)$ \\
$4.29 \pm 0.12(n=323)$ \\
$17.8 \pm 4.3$ \\
$5.1 \pm 1.1$ \\
$3.0 \pm 1.1(n=346)$ \\
26.1 \\
28.7 \\
9.9 \\
2.1 \\
$77.0 \pm 5.0$
\end{tabular}

\begin{tabular}{c} 
Refugee status \\
\hline 2. AFR $(n=175)$ \\
\hline $75.0 \pm 1.4(n=174)$ \\
$9.01 \pm 0.13$ \\
$0.917 \pm 0.023(n=172)$ \\
$4.46 \pm 0.18(n=156)$ \\
$25.4 \pm 8.7$ \\
$4.1 \pm 1.6$ \\
$3.7 \pm 1.9(n=160)$ \\
26.8 \\
31.4 \\
13.6 \\
$n / a$ \\
$79.1 \pm 7.4$
\end{tabular}

3. Native $(n=42,597)$
$66.0 \pm 0.1(n=41,826)$
$8.19 \pm 0.01$
$0.865 \pm 0.001(n=41,83$
$4.88 \pm 0.01(n=38,876)$
$11.5 \pm 0.3$
$2.6 \pm 0.1$
$2.0 \pm 0.1(n=41,554)$
30.6
25.3
6.5
0.2
$53.1 \pm 0.4$

\begin{tabular}{|c|c|c|}
\hline \multicolumn{3}{|c|}{$P$ value } \\
\hline 1 vs 2 & 1 vs 3 & 2 vs 3 \\
\hline ns & $<0.001$ & $<0.001$ \\
\hline 0.02 & $<0.001$ & ns \\
\hline ns & $<0.001$ & ns \\
\hline ns & ns & 0.045 \\
\hline ns & 0.006 & ns \\
\hline ns & ns & ns \\
\hline ns & ns & ns \\
\hline ns & ns & ns \\
\hline ns & 0.039 & 0.002 \\
\hline $\mathrm{n} / \mathrm{a}$ & $<0.001$ & $\mathrm{n} / \mathrm{a}$ \\
\hline ns & $<0.001$ & $<0.001$ \\
\hline
\end{tabular}

Adjusted means with S.E.M. from multivariable regression models with adjustments for age, sex and diabetes duration. The method of Tukey-Kramer was used to adjust for multiple comparisons.

AFR, Africa; DKA, diabetic ketoacidosis; ME, Middle East; pat. yrs., patient years; SMBG, self-monitoring of blood glucose.

with diabetes diagnosis prior to migration to Germany/ Austria, although statistical significance is lacking (13.7 vs $5.3 \%, P=0.18$ ). Intensified insulin therapy was the most common treatment in all groups, independent from the place of diabetes diagnosis (diabetes diagnosis in Germany/Austria vs diabetes diagnosis prior to migration, ME: 76.6 vs $85.4 \%, P=0.15$; AFR: 88.1 vs $78.5 \%, P=0.22$ ).

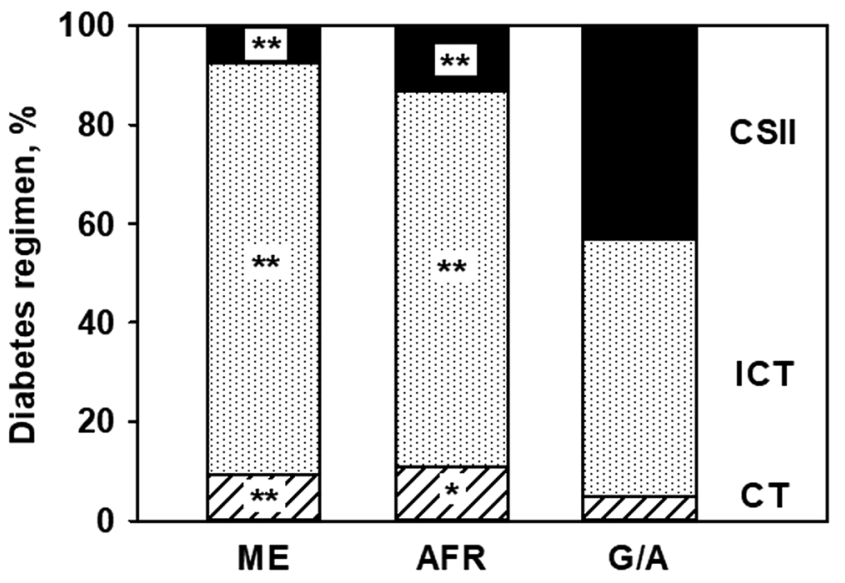

\section{Figure 1}

Treatment regimen in pediatric type 1 diabetes patients, stratified by refugee status. AFR, Africa; CSII, continuous subcutaneous insulin infusion; $C T$, conventional insulin therapy; G/A, Germany/Austria; ICT, intensified insulin therapy; $\mathrm{ME}$, Middle East. Adjusted proportions from multivariable regression models with adjustments for sex, age and diabetes duration. ${ }^{* *} P<0.001$ and ${ }^{*} P<0.01$ for the comparison between refugees (ME/AFR) and native children (G/A).
In African refugees, conventional insulin therapy was documented slightly more frequently if diabetes diagnosis was in Germany or Austria ( 7.6 vs $4.9 \%, P=0.52$ ), whereas no difference was observed for refugees from the ME ( 8.6 vs $8.4 \%, P=0.96)$.

\section{Discussion}

This is the first report on pediatric refugees from Africa or the ME diagnosed with diabetes, describing the first year of diabetes care after arrival in Germany or Austria, and based on a large well-established diabetes registry. Compared to native type 1 diabetes patients, significant differences in metabolic control, diabetes treatment and outcome among refugees from Africa or the ME were observed. This may suggests an increased need for intensified, culturesensitive diabetes care in this particular vulnerable group of patients.

Despite overall higher costs for the health care system due to inadequate access to health care (9), previous articles still criticize restrictions for asylum seekers in Germany $(10,11,12)$. Insufficient knowledge of caregivers on the grant of health care services limits refugees' access to health care (12). On the other hand, language barriers, different health beliefs, limited transport and mobility to specialized clinics and unawareness of national health system procedures may also challenge adequate diabetes care for refugee children. In the Netherlands, frequent relocations, unknown medical history and cultural differences were 
reported by pediatricians as the most frequent barriers for delivering health care (13). Recently, low diabetes literacy and suboptimal diabetes disease control among Somali patients in a US primary care setting have been reported (14). Altogether, may contribute to the worse diabetes control and higher rates of severe hypoglycemia or hypoglycemia with coma in pediatric type 1 diabetes refugees from Africa or ME, compared to native children, as observed in our study. A large number of studies previously reported an association between migration and poor health care outcomes $(5,6,15,16)$ and mentioned different self-care behavior and an overall lower adherence to treatment regimen compared to the native population as potential reasons $(5,17)$. Life style and eating habits often differ between refugees and the host population and further challenge diabetes care for the patient and the caregiver. Apart from that, as reported by Bergenstal et al., 'racial' differences in the glycation of hemoglobin partially explains HbA1c differences between African Americans and whites (18). Although clear evidence is lacking, non-glycemic hemoglobin-related factors such as red blood cell turnover are also speculated to explain ethnic differences in HbA1c (19).

In developed countries, CSII is frequently used among children and adolescents with type 1 diabetes (20). Data on CSII use in Africa or the ME are lacking. In our study, we observed a markedly lower use of modern diabetes technology such as insulin pumps in refugee children from Africa or the ME. As hypothesized by us for other migrant groups from Turkey, Southern Europe or Eastern Europe $(4,5)$, the lower use might be due to the high costs, lack of health care insurance and the complexity and the necessity of intensive diabetes education. Other possible contributors could be different therapeutic concepts in the home country and a potentially lower rate of acceptance of new techniques or insufficient knowledge about health care offers $(4,5)$. Moreover, clinicians' individual assessment whether the patient will manage a complex diabetes regimen may play a role. The uncertain procedure for granting the right of asylum may further complicate CSII initiation or other long-standing therapy decisions in newly arrived refugees.

Besides acute diabetes complications, we also observed long-term complications such as microalbuminuria or diabetic retinopathy that were more prevalent in refugees from Africa or ME compared to native children. A worse metabolic control prior to migration and an inadequate diabetes care in the home country might be possible explanations. Apart from that, there is increasing evidence of a variety of polymorphisms that differ between populations and influence susceptibility to both, retinopathy and nephropathy (21).

Major strengths of our study are the relatively large number of pediatric diabetes cases among refugees from Africa and the $\mathrm{ME}$, and the availability of data from a standardized diabetes patient registry. The registry comprises pre-defined demographic and clinical diabetes-specific parameters that are not specifically adapted to the current situation of diabetes care in refugees. Nevertheless, some weaknesses should be kept in mind. A considerable number of pediatric refugees with diabetes might be missed due to limited/unknown access to specialized diabetes clinics or undiagnosed diabetes. Additional, potentially important individuallevel variables such as mental illnesses or diabetes distress that might explain disparities among refugees and the host population were not available. A cross-sectional German pilot study found a high prevalence of mental illnesses (13.7\%) among unaccompanied asylum-seeking adolescents (22). Depressive symptoms and diabetes distress were reported to be higher in ethnic minorities from Turkey or Morocco compared to native type 1 diabetes patients in the Netherlands (23). Furthermore, unaccompanied children often live in nursing homes that cannot provide adequate support for the diabetes management (24). As our data do not include the family situation we are not able to differentiate this particular group. Lastly, underestimation due to under-reporting of for example hypoglycemic events might bias our findings.

In summary, our report indicates that a relevant number of pediatric refugees from Africa and the ME with type 1 diabetes are treated in German/Austrian specialized diabetes clinics. Compared to native patients, diabetes care and outcome of these children is currently suboptimal. Pediatric refugees, their parents and pediatric diabetes teams are faced with several challenges. The study may suggest the need for additional adaptations in the health care system and clinical practice to target this particularly vulnerable population in order to improve diabetes control and to prevent diabetes complications in the future.

Declaration of interest

The authors declare that there is no conflict of interest that could be perceived as prejudicing the impartiality of this study. 


\section{Funding}

The DPV initiative is supported by the Federal Ministry of Education and Research within the German Center for Diabetes Research (DZD; grant number: 82DZD0017G); the German Robert Koch Institute (RKI); the European Foundation for the Study of Diabetes (EFSD); the German Diabetes Association (DDG) and the Innovative Medicines Initiative 2 Joint Undertaking (INNODIA; grant number: 115797) supported by the European Union's Horizon 2020 research and innovation program and 'EFPIA', 'JDRF' and 'The Leona M. and Harry B. Helmsley Charitable Trust'. The study sponsor was not involved in the design of the study; the collection, analysis and interpretation of data; writing the report or the decision to submit the report for publication.

\section{Author contribution statement}

$\mathrm{N} \mathrm{P}$ and $\mathrm{K} \mathrm{K}$ wrote the manuscript, edited the manuscript, substantial contribution to conception and design and interpretation of data; $\mathrm{C}$ B, E H, A H, A I, J G-H, N J, C K, K M, O R, C S-S and M W researched data, contributed to discussion, reviewed and edited manuscript; $\mathrm{R} \mathrm{W} \mathrm{H}$ conceptualized the study, contributed to discussion, reviewed and edited the manuscript and is the principal investigator of the DPV initiative and as such has full access to the data. All authors approved the final version to be published.

\section{Acknowledgments}

Special thanks to Dr S Lanzinger, MSc Epidemiology, University of Ulm for statistical analyses. The authors also thank A Hungele and R Ranz for DPV documentation software support, and K Fink and E Bollow for DPV data management support (all clinical data managers, University of UIm). Finally, the authors express our gratitude to all participating DPV centers for contributing anonymized data to the present study.

\section{References}

1 European Commission. Eurostat. Where do asylum applicants come from? 2018. (available at: www.ec.europa.eu/eurostat/statisticsexplained/index.php?title=Asylum_quarterly_report\#Where_do_ asylum_applicants_come_from.3F). Accessed on 03 July 2018.

2 Williams B, Cassar C, Siggers G \& Taylor S. Medical and social issues of child refugees in Europe. Archives of Disease in Childhood 2016101 839-842. (https://doi.org/10.1136/archdischild-2016-310657)

3 Hjern A, Østergaard LS, Norredam M, de Luna CM \& Goldfeld S. Health policies for migrant children in Europe and Australia. Lancet 2017389 249. (https://doi.org/10.1016/S0140-6736(17)30084-3)

4 Icks A, Razum O, Rosenbauer J, Bächle C, Hungele A, Mönkemöller K, Müller-Godeffroy E, Heidtmann B, Kapellen T, Scheuing N et al. Lower frequency of insulin pump treatment in children and adolescents of Turkish background with type 1 diabetes: analysis of 21,497 patients in Germany. Diabetes Technology \& Therapeutics 2012 14 1105-1109. (https://doi.org/10.1089/dia.2012.0138)

5 Scheuing N, Wiegand S, Bächle C, Fröhlich-Reiterer E, Hahn E, Icks A, Ludwig KH, Mönkemoller K, Razum O, Rosenbauer J et al. Impact of maternal country of birth on type-1-diabetes therapy and outcome in 27,643 children and adolescents from the DPV Registry. PLOS ONE 201510 e0135178. (https://doi.org/10.1371/journal. pone.0135178)

6 Rosenbauer J, Dost A, Karges B, Hungele A, Stahl A, Bächle C, Gerstl EM, Kastendieck C, Hofer SE, Holl RW et al. Improved metabolic control in children and adolescents with type 1 diabetes: a trend analysis using prospective multicenter data from Germany and Austria. Diabetes Care 201235 80-86. (https://doi.org/10.2337/ dc11-0993)
7 Rosario AS, Kurth BM, Stolzenberg H, Ellert U \& Neuhauser H. Body mass index percentiles for children and adolescents in Germany based on a nationally representative sample (KiGGS 2003-2006). European Journal of Clinical Nutrition 201064 341-349. (https://doi. org/10.1038/ejcn.2010.8)

8 Neuhauser HK, Thamm M, Ellert U, Hense HW \& Rosario AS. Blood pressure percentiles by age and height from non-overweight children and adolescents in Germany. Pediatrics 2011127 e978-e988. (https:// doi.org/10.1542/peds.2010-1290)

9 Bozorgmehr K \& Razum O. Effect of restricting access to health care on health expenditures among asylum-seekers and refugees: a quasi-experimental study in Germany, 1994-2013. PLoS ONE 2015 10 e0131483. (https://doi.org/10.1371/journal.pone.0131483)

10 Bozorgmehr K \& Razum O. Refugees in Germany-untenable restrictions to health care. Lancet $2016 \mathbf{3 8 8} 2351-2352$. (https://doi. org/10.1016/S0140-6736(16)32131-6)

11 Bozorgmehr K, Wenner J \& Razum O. Restricted access to health care for asylum-seekers: applying a human rights lens to the argument of resource constraints. European Journal of Public Health 201727 592-593. (https://doi.org/10.1093/eurpub/ckx086)

12 Razum O, Wenner J \& Bozorgmehr K. When chance decides about access to health care: the case of refugees in Germany. Gesundheitswesen 201678 711-714. (https://doi org/10.1055/s-0042-116231)

13 Baauw A, Rosiek S, Slattery B, Chinapaw M, van Hensbroek MB, van Goudoever JB \& Kist-van Holthe J. Pediatrician-experienced barriers in the medical care for refugee children in the Netherlands. European Journal of Pediatrics 2018177 995-1002. (https://doi. org/10.1007/s00431-018-3141-y)

14 Njeru JW, Hagi-Salaad MF, Haji H, Cha SS \& Wieland ML. Diabetes health literacy among Somali patients with diabetes mellitus in a US primary care setting. Journal of Racial and Ethnic Health Disparities 20163 210-216. (https://doi.org/10.1007/ s40615-015-0129-4)

15 Wieland ML, Morrison TB, Cha SS, Rahman AS \& Chaudhry R. Diabetes care among Somali immigrants and refugees. Journal of Community Health 201237 680-684. (https://doi.org/10.1007/ s10900-011-9499-7)

16 Redondo MJ, Libman I, Cheng P, Kollman C, Tosur M, Gal RL, Bacha F, Klingensmith GJ, Clements M \& Pediatric Diabetes Consortium. Racial/Ethnic minority youth with recent-onset type 1 diabetes have poor prognostic factors. Diabetes Care $2018 \mathbf{4 1}$ 1017-1024. (https://doi.org/10.2337/dc17-2335)

17 Icks A, Rosenbauer J, Strassburger K, Grabert M, Giani G \& Holl RW. Persistent social disparities in the risk of hospital admission of paediatric diabetic patients in Germany-prospective data from 1277 diabetic children and adolescents. Diabetic Medicine 200724 440-442. (https://doi.org/10.1111/j.1464-5491.2007.02105.x)

18 Bergenstal RM, Gal RL, Connor CG, Gubitosi-Klug R, Kruger D, Olson BA, Willi SM, Aleppo G, Weinstock RS, Wood J et al. Racial differences in the relationship of glucose concentrations and hemoglobin A1c levels. Annals of Internal Medicine 2017 167 95-102. (https://doi.org/10.7326/M16-2596)

19 Selvin E. Are there clinical implications of racial differences in HbA1c? A difference, to be a difference, must make a difference. Diabetes Care 201639 1462-1467. (https://doi.org/10.2337/dc160042)

20 Bohn B, Karges B, Vogel C, Otto KP, Marg W, Hofer SE, FröhlichReiterer E, Holder M, Plamper M, Wabitsch M et al. 20 years of pediatric benchmarking in Germany and Austria: age-dependent analysis of longitudinal follow-up in 63,967 children and adolescents with type 1 diabetes. PLOS ONE 201611 e0160971. (https://doi. org/10.1371/journal.pone.0160971)

21 Borchers AT, Uibo R \& Gershwin ME. The geoepidemiology of type 1 diabetes. Autoimmunity Reviews 20109 A355-A365. (https://doi. org/10.1016/j.autrev.2009.12.003) 
22 Marquardt L, Krämer A, Fischer F \& Prüfer-Krämer L. Health status and disease burden of unaccompanied asylum-seeking adolescents in Bielefeld, Germany: cross-sectional pilot study. Tropical Medicine \& International Health 201621 210-218. (https://doi.org/10.1111/ tmi.12649)

23 Schmidt CB, Potter van Loon BJ, Torensma B, Snoek FJ \& Honig A. Ethnic minorities with diabetes differ in depressive and anxiety symptoms and diabetes-distress. Journal of Diabetes Research 2017 2017 1204237. (https://doi.org/10.1155/2017/1204237)

24 Tangermann J \& Hoffmeyer-Zlotnik P. Unaccompanied minors in Germany. 2018. (available at: www.bamf.de/SharedDocs/ Anlagen/DE/Publikationen/EMN/Studien/wp80-unbegleiteteminderjaehrige.pdf?_blob=publicationFile). Accessed on 23 October 2018 .

Received 19 December 2018

Revised version received 15 April 2019

Accepted 10 May 2019 